\section{Case Reports in Ophthalmology}

\title{
A Case of Syphilitic Uveitis in Which Vitreous Surgery Was Useful for the Diagnosis and Treatment
}

\author{
Takatoshi Kobayashi $^{a}$ Chihiro Katsumura ${ }^{a}$ Hiromi Shoda $^{a}$ \\ Nanae Takai ${ }^{a}$ Sayako Takeda, ${ }^{a}$ Takako Okamoto ${ }^{a}$ \\ Koichi Maruyama ${ }^{a, c}$ Rei Tada ${ }^{a, d}$ Kensuke Tajiri ${ }^{a}$ Teruyo Kida ${ }^{a}$ \\ Tsunehiko Ikeda ${ }^{a}$ \\ ${ }^{a}$ Department of Ophthalmology, Osaka Medical College, Takatsuki City, Japan; ${ }^{\mathrm{b}}$ Takeda \\ Eye Clinic, Osaka City, Japan; ${ }^{\mathrm{C}}$ Maruyama Eye Clinic, Takatsuki City, Japan; ${ }^{\mathrm{d}}$ Tada Eye \\ Clinic, Ikeda City, Japan
}

\section{Keywords}

Syphilitic uveitis · Retinal vasculitis · Proliferative retinopathy · Vitreous hemorrhage · Vitreous surgery

\begin{abstract}
Purpose: To report a case of atypical syphilitic uveitis complicated with retinal vasculitis, proliferative retinopathy, and vitreous hemorrhage in which vitreous surgery was useful for the diagnosis and treatment. Case Report: A 38-year-old female was referred to our hospital after noticing visual disturbance in her right eye. Fundoscopy examination of that eye revealed retinal phlebitis accompanied by retinal hemorrhage and soft exudate, and remarkable exudative changes in the retinal vessels from the upper arcade to the macula region. After a blood examination, a serological test showed positive for syphilis; however, systemic findings were scarce. Syphilitic uveitis was suspected, so we administered treatment for syphilis, anticoagulant treatment for retinal vasculitis, steroids for intraocular inflammation, and photocoagulation for the retinal nonperfusion area. However, her visual acuity (VA) decreased to $30 \mathrm{~cm} /$ counting fingers due to vitreous hemorrhage resulting from fibrovascular membrane at the optic disc. Since the vitreous hemorrhage was insufficiently absorbed, vitreous surgery was performed to remove the hemorrhage and fibrovascular tissue. Following
\end{abstract}


surgery, the uveitis and retinal vasculitis subsided, and her corrected VA improved to 0.3. Postoperative examination of a fixed quantity of collected vitreous fluid for syphilis showed a Treponema pallidum hemagglutination value of 5,120 times the normal amount, thus confirming the syphilitic uveitis diagnosis. Conclusions: Our findings show that when observing patients with obstructive retinal vasculitis of unknown causes, syphilitic uveitis should be considered as a differential diagnosis, and that vitreous surgery is useful for the diagnosis and treatment of atypical syphilitic uveitis which has progressed to proliferative retinopathy.

(C) 2017 The Author(s)

Published by S. Karger AG, Basel

\section{Introduction}

Syphilitic uveitis is generally seen in cases of second- or third-stage syphilis; however, the clinical conditions can vary and there are few characteristic findings. In such cases, posterior lesions are generally considered to be chorioretinitis, though with a salt-and-pepper fundus are well known to indicate subsided choroiditis [1, 2]. In this study, we present a case in which vitreous surgery was found useful for the diagnosis and treatment of atypical syphilitic uveitis complicated with extensive ischemia from retinal vasculitis, proliferative retinopathy, and vitreous hemorrhage.

\section{Case Report}

This study involved a 38-year-old female who was followed up by her previous ophthalmologist from 1 month prior to presentation for decreased vision in her right eye. Examination revealed retinal phlebitis in that eye, and she was subsequently referred to our department at Osaka Medical College Hospital in order to obtain a more detailed examination and medical treatment.

\section{Medical History}

Nothing notable was observed in the patient's medical history.

\section{Initial Medical Examination Findings}

In the patient's right and left eye, respectively, visual acuity (VA) was $0.04(0.05 \times \mathrm{s}-$ $1.75 \mathrm{dpt})$ and $0.3\left(1.5 \times \mathrm{s}-1.25 \mathrm{dpt}=\right.$ cylinder $-0.5 \mathrm{dpt}$, axis $\left.10^{\circ}\right)$, intraocular pressure was $10 \mathrm{~mm} \mathrm{Hg}$ and $12 \mathrm{~mm} \mathrm{Hg}$, and no abnormality of the anterior segment was observed. In the right eye, we observed a few inflammatory cells in the anterior chamber, retinal phlebitis accompanied by retinal hemorrhage and soft exudate, and remarkable exudative changes in the retinal vessels from the upper arcade to the macula region (Fig. 1a). Ophthalmoscopy also revealed obvious cystoid macular edema. No abnormalities in the left eye fundus were observed (Fig. 1b). Fluorescein angiography revealed obstructive retinal vasculitis along the upper vascular arcade of the right eye. A general health examination showed a positive tuberculin skin test result; however, a chest X-ray of the patient showed no abnormalities. A general blood examination revealed a positive rapid plasma regain syphilis serology test result, positive results for Treponema pallidum antibodies (rapid plasma regain 8-fold, $T$. pallidum antibodies 8.8 cutoff index), and positive results for fluorescent treponemal antibody absorption immunoglobulin $\mathrm{M}$ antibodies. Testing for collagen disease revealed positive results for anticardiolipin antibodies, yet there were no abnormal neurological findings. 


\section{Case Reports in Ophthalmology}

\begin{tabular}{l|l}
\hline DOI: $10.1159 / 000455910$ & (c) 2017 The Author(s). Published by S. Karger AG, Basel \\
\hline
\end{tabular} www.karger.com/cop

Kobayashi et al.: A Case of Syphilitic Uveitis in Which Vitreous Surgery Was Useful for the Diagnosis and Treatment

A magnetic resonance imaging examination of the patients head revealed no abnormal findings, yet dermatological findings included a painless rash on the pudenda of 4 months previously. Other than that, no syphilis-related skin conditions were found.

\section{Treatment Progress}

Administration of anticoagulants was initiated to treat the retinal vasculitis. However, triamcinolone acetonide was injected into the Tenon capsule at 3 weeks after the start of treatment due to worsening of the vasculitis. Moreover, administration of a broad-spectrum penicillin preparation was started to treat the syphilis infection. However, at 50 days after the initial examination, new neovascularization appeared from the optic disc to the upper retinal vascular arcade, causing vitreous hemorrhage (Fig. 2a), and the corrected VA of the patient's right eye decreased to 0.02 . Fluorescein angiography revealed dye leakage from the disc neovascularization and enlargement of the nonperfusion area in the affected retina (Fig. $2 b)$. At 3 months after the initial examination, photocoagulation was performed on the nonperfusion area of the retina. However, no regression of the neovascularization was observed, and vitreous rebleeding occurred. At 4 months after the initial examination, vitreous surgery was performed because the patient's corrected VA had worsened to counting fingers. Surgical procedures included a core vitrectomy, creation of an artificial posterior detachment from the optic disc to the peripheral region, removal of the fibrovascular membrane from the optic disc to the upper retinal vascular arcade, and laser endophotocoagulation to the ischemic area of the retina. Since the patient was only 38 years old (i.e., relatively young), the lens was preserved. A postoperative syphilis test of a fixed quantity of collected vitreous humor showed a glass plate test value of 1 , and a T. pallidum hemagglutination (TPHA) value of 5,120 . Simultaneously, serum from collected blood showed a glass plate test value of 2 and a TPHA value of 640 , thus confirming the diagnosis of syphilitic uveitis. At 3 months postoperatively, the intraocular neovascularization had disappeared (Fig. 3a), the fluorescein leakage from the retinal blood vessels had clearly decreased (Fig. 3b), and the corrected VA had improved to 0.3 .

\section{Discussion}

In cases of syphilitic uveitis, T. pallidum organizes within the eye via blood flow and results in inflammation in $0.3-2 \%$ of syphilis recenta cases. Clinical findings are varied, yet generally include iridocyclitis, chorioretinitis, retinal pigment epitheliitis, serous retinal detachment, cystoid macular edema, vitreous opacity, and neuroretinitis, etc. [1, 2]. Although there are few characteristic findings, the most common type of syphilitic uveitis is chorioretinitis, in which infiltration of inflammatory cells from the choroid capillaries, afflicting the Bruch's membrane/retinal pigment epithelium, is thought to constitute the pathological progression. This present case had remarkable retinal phlebitis, and fluorescein angiography revealed leakage and staining of the retinal vein, as well as an extensive retinal nonperfusion area. Moreover, there was a massive vitreous hemorrhage caused by neovascularization of the optic disc, thus necessitating vitreous surgery. These findings in the present case suggest that the primary pathological locations were the optic nerve and retinal blood vessels, which are different from the clinical findings in typical syphilitic uveitis cases.

In recent years, it has been reported that atypical clinical findings of syphilitic uveitis, such as in this present case, are sometimes accompanied by human immunodeficiency virus (HIV) infection [3, 4]. At present, and more than in previous years, the frequency of syphilitic 


\section{Case Reports in Ophthalmology}

Case Rep Ophthalmol 2017;8:55-60

DOI: 10.1159/000455910

(c) 2017 The Author(s). Published by S. Karger AG, Basel www.karger.com/cop

Kobayashi et al.: A Case of Syphilitic Uveitis in Which Vitreous Surgery Was Useful for the Diagnosis and Treatment

uveitis in HIV-infected individuals is significantly higher than that in uninfected individuals, and it is now known that it can easily develop from the early stages of neurosyphilis. In this present case, no findings were observed that led to a suspicion of HIV infection or neurosyphilis. However, it is thought that the optic nerve and retina are the chief locations in which neurosyphilis can form [5-8]. Yokoi and Kase [9] considered retinal vasculitis to be one symptom of optical syphilis, with retinal blood vessel obstructions occurring simultaneously in the arteries, veins, and capillaries, thus causing irreversible changes in the vascular walls. In addition, Zhang et al. [3] posited that retinal vasculitis and optic neuritis are not rare findings in cases of syphilitic uveitis.

As a differential diagnosis for this disease, we first theorized retinal vasculitis in connection with collagen disease. Behçet disease was ruled out due to the absence of clear general findings such as oral aphthae, genital ulcer, and erythema nodosum. Moreover, sarcoidosis was ruled out due to the absence of general findings, as was systemic lupus erythematosus due to the negative antinuclear antibody results. Since the anticardiolipin antibody tests were positive, antiphospholipid antibody syndrome was considered a possibility. However, it was ruled out due to the fact that no corresponding findings, other than the antinuclear antibody positivity, were present.

Next, retinal vasculitis due to infection was theorized. Retinal vasculitis resulting from HIV was ruled out due to the negative HIV antibody results, and thus an absence of immunodeficiency. Fundus findings suggested the possibility of tuberculous uveitis, which was ruled out due to no tuberculosis findings being present in the chest X-ray that was given to the patient as part of the initial comprehensive examination. At first, syphilitic uveitis could not be definitively diagnosed from the general findings, other than the positive serological test result for syphilis. However, the abnormally high TPHA value of 5,120 in the vitreous fluid collected during vitreous surgery, as well as the TPHA value of 640 in the serum of blood collected at the same time, confirmed the diagnosis.

Treatment for syphilitic uveitis commonly includes administration of large doses of penicillin to treat the syphilis, while simultaneously providing local steroid treatment to the ocular inflammation $[1,2]$. In addition, in cases such as the one described in this study, when an outbreak of neovascularization from an extensive retinal nonperfusion area occurs, fluorescein angiography and photocoagulation should be performed in the early stages. When photocoagulation proves ineffective and the vitreous hemorrhage and proliferative retinopathy continue to progress, vitreous surgery is indicated.

There have been previous reports of vitreous surgery for syphilitic uveitis in which vitreous opacity was a common indication. Among them can be seen cases like this one in which vitreous surgery was effective for proliferative changes from the obstructive retinal vasculitis. Queiroz et al. [10] reported 4 cases in which proliferative retinopathy developed in the early stages following syphilis treatment and for which vitreous surgery was performed, and they identified a high rate of retinal necrotic change. Haug et al. [11] report 11 cases of rhegmatogenous retinal detachment complicated with syphilitic uveitis, theorizing that retinal necrosis caused by the syphilis was the origin of the tear formation. In cases of fulminant syphilitic uveitis, such as that described in this present study, in addition to proliferative alterations brought about by retinal ischemia, it is important to also be cautious of rhegmatogenous retinal detachment caused by retinal necrosis.

As in the case presented here, upon seeing obstructive retinal vasculitis caused by proliferative retinopathy of an unknown origin, syphilitic uveitis should also be kept in mind as a potential primary disease. Prompt and appropriate treatment is necessary, with a thorough general examination in the early stages. Treatment for syphilis should be administered 
as necessary, combined with anti-inflammatory treatment, anticoagulation treatment, photocoagulation, and vitreous surgery, etc., when suitable. Moreover, in cases such as the one described here in which general findings are meager, and in which a definitive syphilis diagnosis is difficult, we believe that serological testing of vitreous samples obtained during vitreous surgery can prove useful in the diagnosis.

\section{Acknowledgment}

The authors wish to thank John Bush for editing the manuscript.

\section{Statement of Ethics}

This case study has been approved by the Ethics Committee of the Osaka Medical College.

\section{Disclosure Statement}

There are no conflicts of interest to report for all authors.

\section{References}

1 Kiss S, Damico FM, Young LH: Ocular manifestations and treatment of syphilis. Semin Ophthalmol 2005;20:161-167.

2 Doris JP, Saha K, Jones NP, Sukthankar A: Ocular syphilis: the new epidemic. Eye (Lond) 2006;20:703705.

3 Zhang R, Qian J, Guo J, Yuan Y, Xue K, Yue H, Chen L: Clinical manifestations and treatment outcomes of syphilitic uveitis in a Chinese population. J Ophthalmol 2016;2016:2797028.

-4 Wickremasinghe S, Ling C, Stawell R, Yeoh J, Hall A, Zamir E: Syphilitic punctate inner retinitis in immunocompetent gay men. Ophthalmology 2009;116:1195-1200.

5 Chebil A, Maamouri R, Ouaderni M, Limaiem R, El Matri L: Syphilitic uveitis revealed by bilateral retinal occlusive vasculitis. Tunis Med 2015;93:653-654.

-6 Yang P, Zhang N, Li F, Chen Y, Kijlstra A: Ocular manifestations of syphilitic uveitis in Chinese patients. Retina 2012;32:1906-1914.

7 Morgan CM, Webb RM, O'Connor GR: Atypical syphilitic chorioretinitis and vasculitis. Retina 1984;4:225-231.

8 Weinstein JM, Lexow SS, Ho P, Spickards A: Acute syphilitic optic neuritis. Arch Ophthalmol 1981;99:1392-1395.

Yokoi M, Kase M: Retinal vasculitis due to secondary syphilis. Jpn J Ophthalmol 2004;48:65-67. Queiroz Rde P, Diniz AV, Vasconcelos-Santos DV: Fulminant proliferative vitreoretinopathy in syphilitic uveitis. J Ophthalmic Inflamm Infect 2016;6:6.

11 Haug SJ, Takakura A, Jumper JM, Heiden D, McDonald HR, Johnson RN, Fu AD, Lujan BJ, Cunningham ET Jr: Rhegmatogenous retinal detachment in patients with acute syphilitic panuveitis. Ocul Immunol Inflamm 2016;24:69-76. 


\section{Case Reports in Ophthalmology}

\begin{tabular}{l|l}
\hline DOI: 10.1159/000455910 & $\begin{array}{l}\text { C } 2017 \text { The Author(s). Published by S. Karger AG, Basel } \\
\text { www.karger.com/cop }\end{array}$ \\
\hline
\end{tabular}

Kobayashi et al.: A Case of Syphilitic Uveitis in Which Vitreous Surgery Was Useful for the Diagnosis and Treatment
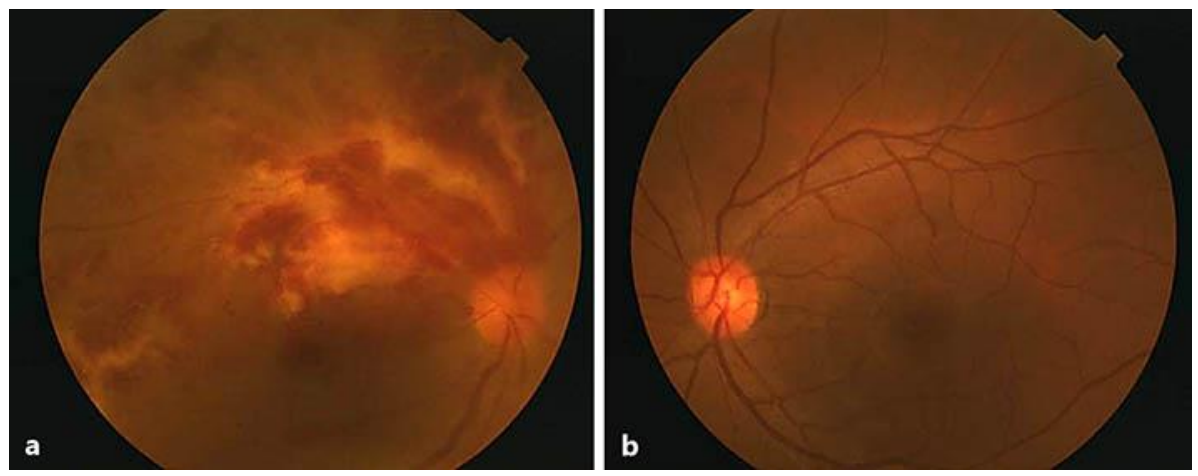

Fig. 1. Funduscopy images of the patient's right eye (a) and left eye (b) obtained at the initial examination. Retinal phlebitis accompanied by retinal hemorrhage, soft exudate, and exudative changes in the retinal vessels from the upper arcade to the macula region can be seen in the right eye (a), while no abnormalities can be seen in the left eye (b).
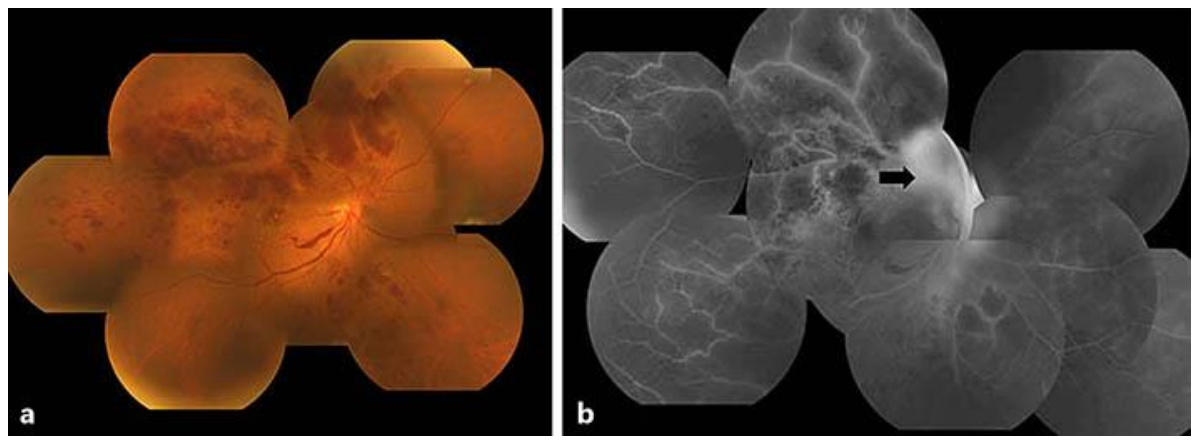

Fig. 2. Funduscopy (a) and fluorescein fundus (b) images obtained at 50 days after the initial examination. Neovascularization that caused vitreous hemorrhage can be seen from the optic disc to the upper retinal vascular arcade. Fluorescein angiography revealed dye leakage from the optic disc neovascularization and fibrovascular membrane (black arrow), and enlargement of the nonperfusion area in the affected retina. The corrected visual acuity of the right eye had decreased to 0.02 .
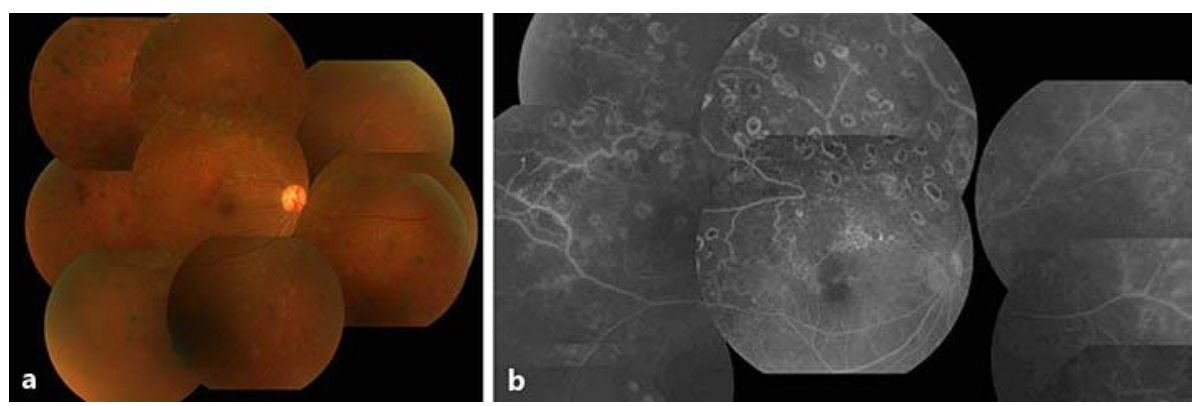

Fig. 3. Funduscopy (a) and fluorescein fundus (b) images obtained at 3 months postoperatively. The neovascularization had disappeared, and the fluorescein leakage from the retinal blood vessels had clearly decreased. The corrected visual acuity of the right eye had improved to 0.3 . 